\title{
Some Results on Point-Line Trajectories in Lorentz 3-space
}

\author{
Tunahan Turhan* Vildan Özdemir and Nihat Ayyıldız \\ (Communicated by Murat Tosun)
}

\begin{abstract}
In this paper, we study curvature theory of point-line trajectories in Lorentz 3-space. We give the characterization by indicatrix, directrix and their relationship in Lorentz 3-space. We use this characterization and relationship to depict a point-line trajectory.
\end{abstract}

Keywords: Curvature; Directrix; Indicatrix; Point-line trajectories; Lorentzian 3-space.

AMS Subject Classification (2010): 53A04 ; 53A25; 53A40.

\section{Introduction}

Point-line trajectory is a very commonly encountered topic in many industrial applications such as welding, cutting, painting, milling, screwing and instrument probing. Point-line trajectory is used as a tool to trace a path with the tool axis oriented in a certain direction while the rotation about the axis is irrelevant, [13].

There are many studies dealing with the curvature theory of line trajectory $[1,2,3,4,5,6$, 7]. One of these studies is presented by McCarthy and Roth [6]. Then, Ryuh and Pennock [10] carried out this theory to trajectory planing of robot end-effectors. They studied directrix and the trajectory of the tool center point. But, the relationship and coordination between the directrix and indicatrix are given by Ting and friends [13]. For detailed information, see $[2,3,4,5,6,7,8,9,10,11,12,13,1]$.

This study is related with relationship and coordination between the directrix and indicatrix in Lorentz 3 -space and presents curvature theory of point-line trajectories in Lorentz 3-space. We investigate Ting and friends', [13], results in Lorentz 3-space.

\section{Setting and Notations}

Let $\mathbb{E}_{1}^{3}$ be Lorentz 3 -space with the inner product

$$
\langle u, v\rangle=-u_{1} v_{1}+u_{2} v_{2}+u_{3} v_{3}
$$

and the vector product

$$
u \times v=\left(-\left|\begin{array}{ll}
u_{2} & u_{3} \\
v_{2} & v_{3}
\end{array}\right|,\left|\begin{array}{cc}
u_{3} & u_{1} \\
v_{3} & v_{1}
\end{array}\right|,\left|\begin{array}{cc}
u_{1} & u_{2} \\
v_{1} & v_{2}
\end{array}\right|\right),
$$

where $u=\left(u_{1}, u_{2}, u_{3}\right), v=\left(v_{1}, v_{2}, v_{3}\right) \in \mathbb{E}_{1}^{3}$, [8].

Definition. A vector $u$ in $\mathbb{E}_{1}^{3}$ is said to be spacelike if $\langle u, u\rangle>0$ or $u=0$, timelike if $\langle u, u\rangle\langle 0$, lightlike or null if $\langle u, u\rangle=0$ and $u \neq 0$. We define the signature of a vector $u$ as

$$
\varepsilon=\left\{\begin{array}{cc}
1, & u \text { is spacelike } \\
0, & u \text { is lightlike } \\
-1, & u \text { is timelike }
\end{array}\right.
$$

The norm of a vector $u \in \mathbb{E}_{1}^{3}$ is defined by $\|u\|=\sqrt{|\langle u, u\rangle|}$, [8]. 


\section{Characterization by Indicatrix, Directrix and Their Relationship}

A point-line trajectory with a one parameter motion is a patch on a ruled surface that can be denoted as a two dimensional set of points defined by the succeding equation

$$
X(t, \psi)=r(t)+\psi R(t)
$$

where $t$ is an independent parameter, $r(t)$ and $R(t)$ are respectively, the directrix and indicatrix of the point-line trajectory and $\psi$ is also an independent parameter indicating a physical point on the point-line determined by $r(t)$ and $R(t)$.

The indicatrix of a point-line trajectory in Lorentz 3-space can be acted as a spherical curve $R(t)$ on a unit pseudo sphere $S_{1}^{2}$ in $\mathbb{E}_{1}^{3}$. Let $s_{R}$ be the arc length of the spherical curve

$$
s_{R}=\int_{0}^{t}\langle\dot{R}, \dot{R}\rangle^{1 / 2} d t
$$

The unit spacelike tangent vector $T$ to the indicatrix is given by the derivative of $R$ with respect to $s_{R}$ is

$$
R^{\prime}=T \text {. }
$$

Let $K=R \times T$ be a timelike vector, the three mutually orthogonal unit vectors $[R, T, K]$ define a geodesic trihedron and denoted by $[R]$. The first order derivative of $[R]$ with respect to $s_{R}$ is

$$
\left[\begin{array}{l}
R^{\prime} \\
T^{\prime} \\
K^{\prime}
\end{array}\right]=\left[\begin{array}{ccc}
0 & 1 & 0 \\
-1 & 0 & \gamma_{R} \\
0 & \gamma_{R} & 0
\end{array}\right]\left[\begin{array}{l}
R \\
T \\
K
\end{array}\right],
$$

where $\gamma_{R}$ is the geodesic curvature.

Unit timelike vector $N$ of $R(t)$ represents the direction of $T^{\prime}$. Let $B=-T \times N$ be a spacelike vector and called binormal of $R(t)$. The three mutually orthogonal unit vectors $[T, N, B]$ define a natural trihedron and denoted by $[T]$. Their derivative relations are given as

$$
\left[\begin{array}{l}
T^{\prime} \\
N^{\prime} \\
B^{\prime}
\end{array}\right]=\left[\begin{array}{ccc}
0 & \kappa_{R} & 0 \\
\kappa_{R} & 0 & \tau_{R} \\
0 & \tau_{R} & 0
\end{array}\right]\left[\begin{array}{l}
T \\
N \\
B
\end{array}\right],
$$

where curvature $\kappa_{R}$ and torsion $\tau_{R}$ characterize completely differential properties of the indicatrix up to the third order. Since $R(t)$ stays on a unit pseudo sphere, from [14], it can be proved that

$$
\tau_{R}= \pm \frac{\kappa_{R}^{\prime}}{\kappa_{R} \sqrt{1 \pm \kappa_{R}^{2}}}
$$

and $\kappa_{R}$ and $\gamma_{R}$ have the following relationship

$$
\kappa_{R}^{2}= \pm\left(\gamma_{R}^{2}-1\right) .
$$

The relationship between geodesic trihedron $[R]$ and natural trihedron $[T]$ can be expressed as

$$
\left[\begin{array}{l}
T \\
N \\
B
\end{array}\right]=\left[\begin{array}{ccc}
0 & 1 & 0 \\
\sinh \theta & 0 & \cosh \theta \\
\cosh \theta & 0 & \sinh \theta
\end{array}\right]\left[\begin{array}{l}
R \\
T \\
K
\end{array}\right],
$$

where $\theta$ is referred to as the hyperbolic angle. (See Figure 1.) From equations (4) and (5), we have

$$
\sinh \theta=-\frac{1}{\kappa_{R}} \text { and } \cosh \theta=\frac{\gamma_{R}}{\kappa_{R}} .
$$

In some cases it may be desirable the derivatives according to $t$. So, the derivatives of $R(t)$ are obtained as

$$
\dot{R}=\frac{d R}{d t}=\gamma T
$$




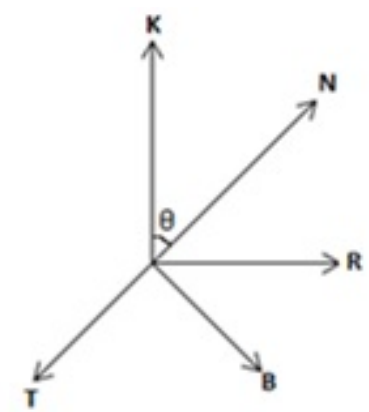

Figure 1. Trihedrons $[R]$ and $[T]$ and the hyperbolic angle

$$
\begin{gathered}
\ddot{R}=\dot{\gamma} T+\gamma^{2} \kappa_{R} N, \\
\dddot{R}=\left(\ddot{\gamma}+\gamma^{3} \kappa_{R}^{2}\right) T+\left(3 \dot{\gamma} \gamma \kappa_{R}+\gamma^{3} \kappa_{R}^{\prime}\right) N+\gamma^{3} \kappa_{R} \tau_{R} B .
\end{gathered}
$$

Here, $\gamma=\frac{d s_{R}}{d t}$ is speed of the indicatrix or the angular velocity of the point-line motion.

The directrix of a point-line trajectory is a spacelike curve denoted by $r(t)$. Let $s_{r}$ refer to the arc length of the directrix,

$$
s_{r}=\int_{0}^{t}\langle\dot{r}, \dot{r}\rangle^{1 / 2} d t
$$

and the derivatives of a variable with respect to $s_{r}$ in the context of directrix be denoted by a superscript prime. The unit spacelike tangent vector $\mathbf{t}$ of the directrix, with respect to $s_{r}$ :

$$
r^{\prime}=\mathbf{t} .
$$

Therefore, the Frenet relations of $r(t)$ can be written as

$$
\left[\begin{array}{l}
\mathbf{t}^{\prime} \\
\mathbf{n}^{\prime} \\
\mathbf{b}^{\prime}
\end{array}\right]=\left[\begin{array}{ccc}
0 & \kappa_{r} & 0 \\
\kappa_{r} & 0 & \tau_{r} \\
0 & \tau_{r} & 0
\end{array}\right]\left[\begin{array}{l}
\mathbf{t} \\
\mathbf{n} \\
\mathbf{b}
\end{array}\right],
$$

where $\mathbf{t}, \mathbf{n}, \mathbf{b}$ are the unit spacelike tangent, timelike normal and spacelike binormal vectors of $r(t)$ and $\kappa_{r}$ and $\tau_{r}$ are the curvature and torsion of $r(t)$. Let us denote the unit vectors $\mathbf{t}, \mathbf{n}$ and $\mathbf{b}$ as $[t]$.

The derivatives of $r(t)$ with respect to $t$ can be expressed as follows;

$$
\begin{gathered}
\dot{r}=\frac{d r}{d t}=v \mathbf{t}, \\
\ddot{r}=\dot{v} \mathbf{t}+v^{2} \kappa_{r} \mathbf{n}, \\
\dddot{r}=\left(\ddot{v}+v^{3} \kappa_{r}^{2}\right) \mathbf{t}+\left(3 v \dot{v} \kappa_{r}+v^{3} \kappa_{r}^{\prime}\right) \mathbf{n}+v^{3} \kappa_{r} \tau_{r} \mathbf{b},
\end{gathered}
$$

where $v=\frac{d s_{r}}{d t}$ is the speed of the directrix.

In the text which follows, we will treat the relationship between the directrix and indicatrix curves.

The directrix and indicatrix have been characterized separately up to now. To completely characterize a pointline trajectory, we must also regard the relationship between the directrix and indicatrix.

The relation between the arc lengths $s_{R}$ and $s_{r}$ is shown by

$$
\xi=\frac{d s_{r}}{d s_{R}},
$$

where $\xi$ is referred to as the velocity ratio. Accordingly, the speed of the directrix and its derivatives can be given as follows

$$
\begin{gathered}
v=\xi \gamma, \\
\dot{v}=\xi^{\prime} \gamma^{2}+\xi \dot{\gamma}, \\
\ddot{v}=\xi^{\prime \prime} \gamma^{3}+3 \xi^{\prime} \gamma \dot{\gamma}+\xi \ddot{\gamma},
\end{gathered}
$$

where $\xi^{\prime}=\frac{d \xi}{d s_{R}}$ and $\xi^{\prime \prime}=\frac{d^{2} \xi}{d s_{R}^{2}}$. 


\section{Coordination of Directrix and Indicatrix}

From the point trajectory, the $\{\mathbf{t}, \mathbf{n}, \mathbf{b}\}$ trihedron and the characteristic numbers characterizing the directrix can be determined. To coordinate the direction of the point-line axis with the indicatrix up to the second order, the required characteristic numbers $a_{\mathbf{t} T}, \kappa_{R}, \xi$, and $\xi^{\prime}$ need to be determined, [13].

The orientation of the point-line axis with respect to the point trajectory is characterized by parameters $a_{\mathbf{t} R}$ and $a_{\mathbf{n} R}$. If such a directional relationship is to remain unchanged, $a_{\mathbf{t} R}$ and $a_{\mathbf{n} R}$ must be constant. Since $a_{\mathbf{t} R}=R$.t and $a_{\mathbf{n} R}=R . \mathbf{n}$ where $R$ is the unit spacelike vector along the point-line axis, taking the derivatives over the conditions that $a_{\mathbf{t} R}$ and $a_{\mathbf{n} R}$ are constant yields

$$
\begin{aligned}
& \dot{R} \cdot \mathbf{t}+R \cdot \dot{\mathbf{t}}=0, \\
& \dot{R} \cdot \mathbf{n}+R \cdot \dot{\mathbf{n}}=0 .
\end{aligned}
$$

From equations (10), (15) and (19) the above two equations can be rewritten as, respectively,

$$
a_{\mathbf{t} T}+\xi \kappa_{r} a_{\mathbf{n} R}=0
$$

and

$$
a_{\mathbf{n} T}+\xi \kappa_{r} a_{\mathbf{t} R}+\xi \tau_{r} a_{\mathbf{b} R}=0 .
$$

Equations (23) and (24) or (25) and (26) are the conditions for the point-line axis to maintain a consistent direction with respect to the directrix up to the first order.

Let $T=\left(T_{x}, T_{y}, T_{z}\right), \mathbf{t}=\left(t_{x}, t_{y}, t_{z}\right), \mathbf{n}=\left(n_{x}, n_{y}, n_{z}\right)$ and if we exchange them into equations (25) and (26), we get

$$
\begin{gathered}
t_{x} T_{x}+t_{y} T_{y}+t_{z} T_{z}+\xi \kappa_{r} a_{\mathbf{n} R}=0, \\
n_{x} T_{x}+n_{y} T_{y}+n_{z} T_{z}+\xi \kappa_{r} a_{\mathbf{t} R}+\xi \tau_{r} a_{\mathbf{b} R}=0 .
\end{gathered}
$$

Unifying the above two equations with the constraints on the magnitude and direction of $T$,

$$
T_{x}^{2}+T_{y}^{2}+T_{z}^{2}=1
$$

and

$$
R . T=0 \text { and } R_{x} T_{x}+R_{y} T_{y}+R_{z} T_{z}=0,
$$

where $R=\left(R_{x}, R_{y}, R_{z}\right), T$ and $\xi$ can be solved. If we solve this equation systems, we get $T$ and $\xi$ as below,

$$
\begin{aligned}
\xi & =\frac{\left|c_{0}\right|}{\sqrt{c_{1}^{2}+c_{2}^{2}+c_{3}^{2}}} \\
T_{x} & = \pm \frac{c_{1}}{\sqrt{c_{1}^{2}+c_{2}^{2}+c_{3}^{2}}}, T_{y}= \pm \frac{c_{2}}{\sqrt{c_{1}^{2}+c_{2}^{2}+c_{3}^{2}}}, T_{z}= \pm \frac{c_{3}}{\sqrt{c_{1}^{2}+c_{2}^{2}+c_{3}^{2}}},
\end{aligned}
$$

where

$$
\begin{aligned}
c_{0}= & -t_{x} n_{z} R_{y}+t_{x} n_{y} R_{z}-t_{y} n_{x} R_{z}+t_{y} n_{z} R_{x}-t_{z} n_{y} R_{x}+t_{z} n_{x} R_{y}, \\
c_{1}= & -a_{\mathbf{t} R} \kappa_{r} R_{y} t_{z}-a_{\mathbf{b} R} \tau_{r} R_{y} t_{z}+a_{\mathbf{n} R} \kappa_{r} R_{y} n_{z}+a_{\mathbf{t} R} \kappa_{r} R_{z} t_{y} \\
& +a_{\mathbf{b} R} \tau_{r} R_{z} t_{y}-a_{\mathbf{n} R} \kappa_{r} R_{z} n_{y} \\
c_{2}= & a_{\mathbf{n} R} \kappa_{r} R_{z} n_{x}+a_{\mathbf{t} R} \kappa_{r} R_{x} t_{z}+a_{\mathbf{b} R} \tau_{r} R_{x} t_{z}-a_{\mathbf{n} R} \kappa_{r} R_{x} n_{z} \\
& -a_{\mathbf{t} R} \kappa_{r} R_{z} t_{x}-a_{\mathbf{b} R} \tau_{r} R_{z} t_{x} \\
c_{3}= & -a_{\mathbf{n} R} \kappa_{r} R_{y} n_{x}-a_{\mathbf{t} R} \kappa_{r} R_{x} t_{y}-a_{\mathbf{b} R} \tau_{r} R_{x} t_{y}+a_{\mathbf{n} R} \kappa_{r} R_{x} n_{y} \\
& +a_{\mathbf{t} R} \kappa_{r} R_{y} t_{x}+a_{\mathbf{b} R} \tau_{r} R_{y} t_{x} .
\end{aligned}
$$

If we take the derivative over equations (23) and (24), we have

$$
\begin{aligned}
& \ddot{R} \cdot \mathbf{t}+2 \dot{R} \cdot \dot{\mathbf{t}}+R . \ddot{\mathbf{t}}=0, \\
& \ddot{R} \cdot \mathbf{n}+2 \dot{R} \cdot \dot{\mathbf{n}}+R \cdot \ddot{\mathbf{n}}=0 .
\end{aligned}
$$


With equations (10), (11), (15) and (19) - (21) the above equations can be rewritten as

$$
\begin{aligned}
& \frac{1}{\xi}\left(\dot{v}-\gamma^{2} \xi^{\prime}\right) a_{\mathbf{t} T}+\gamma^{2} \kappa_{R} a_{\mathbf{t} N}+2 \gamma v \kappa_{r} a_{\mathbf{n} T}+\left(\dot{v} \kappa_{r}+v^{2} \kappa_{r}^{\prime}\right) a_{\mathbf{n} R}+v^{2} \kappa_{r}^{2} a_{\mathbf{t} R} \\
& \quad+v^{2} \kappa_{r} \tau_{r} a_{\mathbf{b} R}=0, \\
& \frac{1}{\xi}\left(\dot{v}-\gamma^{2} \xi^{\prime}\right) a_{\mathbf{n} T}+\gamma^{2} \kappa_{R} a_{\mathbf{n} N}+2 \gamma v\left(\kappa_{r} a_{\mathbf{t} T}+\tau_{r} a_{\mathbf{b} T}\right)+\dot{v}\left(\kappa_{r} a_{t R}+\tau_{r} a_{\mathbf{b} R}\right) \\
& \quad+v^{2}\left(\kappa_{r}^{\prime} a_{\mathbf{t} R}+\kappa_{r}^{2} a_{\mathbf{n} R}+\tau_{r}^{\prime} a_{\mathbf{b} R}+\tau_{r}^{2} a_{\mathbf{n} R}\right)=0 .
\end{aligned}
$$

$\kappa_{R}$ and $\xi^{\prime}$ can be solved from the above two equations. If we solve these equations, we get

$$
\xi^{\prime}=\frac{\left|\begin{array}{ll}
c_{6} & c_{5} \\
c_{9} & c_{8}
\end{array}\right|}{\left|\begin{array}{ll}
c_{4} & c_{5} \\
c_{7} & c_{8}
\end{array}\right|} \text { and } \kappa_{R}=\frac{\left|\begin{array}{ll}
c_{4} & c_{6} \\
c_{7} & c_{9}
\end{array}\right|}{\left|\begin{array}{ll}
c_{4} & c_{5} \\
c_{7} & c_{8}
\end{array}\right|}
$$

where

$$
\begin{aligned}
c_{4}= & -\frac{\gamma^{2} a_{\mathbf{t} T}}{\xi}, c_{5}=\gamma^{2} a_{\mathbf{t} N}, \\
c_{6}= & -\frac{\dot{v} a_{\mathbf{t} T}}{\xi}-2 \gamma v \kappa_{r} a_{\mathbf{n} T}-\left(\dot{v} \kappa_{r}+v^{2} \kappa_{r}^{\prime}\right) a_{\mathbf{n} R}-v^{2} \kappa_{r}^{2} a_{\mathbf{t} R}-v^{2} \kappa_{r} \tau_{r} a_{\mathbf{b} R}, \\
c_{7}= & -\frac{\gamma^{2} a_{\mathbf{n} T}}{\xi}, c_{8}=\gamma^{2} a_{\mathbf{n} N}, \\
c_{9}= & -\frac{\dot{v} a_{\mathbf{n} T}}{\xi}-2 \gamma v\left(\kappa_{r} a_{\mathbf{t} T}+\tau_{r} a_{\mathbf{b} T}\right)-\dot{v}\left(\kappa_{r} a_{t R}+\tau_{r} a_{\mathbf{b} R}\right) \\
& -v^{2}\left(\kappa_{r}^{\prime} a_{\mathbf{t} R}+\kappa_{r}^{2} a_{\mathbf{n} R}+\tau_{r}^{\prime} a_{\mathbf{b} R}+\tau_{r}^{2} a_{\mathbf{n} R}\right) .
\end{aligned}
$$

\section{Conclusions}

This paper gives the properties of the curvature theory of point-line trajectories in Lorentzian 3-space. We have shown that the relation between the curvature and geodesic curvature of indicatrix. The relation between the direction and indicatrix curves are given.

\section{References}

[1] Aydoğmuş, Ö., Kula, L. and Yaylı, Y., On point-line displacement in Minkowski 3-space. Differential Geometry-Dynamical Systems, 10 (2008), 32-43.

[2] Bottema, O., and Roth, B., Theoretical Kinematics, Dover Publications, New York, 1990.

[3] Hunt, K. H., Kinematic Geometry of Mechanisms, Clarendon Press, Oxford, England, 465 p., 1978.

[4] Kirson, Y., Higher order curvature theory in space kinematics. Ph.D dissertation, University of California at Berkeley, 140 p., 1975.

[5] Kim, J. H., Ruyh, B. S., and Pennock, G. R., Development of a Trajectory Generation Method for a Five-Axis NC Machine. Mech. Mach. Theory, 36 (2001), 983-996.

[6] McCarthy, J. M., and Roth, B., The Curvature Theory of Line Trajectories in Spatial Kinematics. ASME J. Mech. Des., 103 (1981), 718-724.

[7] McCarthy, J. M., The Instantaneous Kinematics of Line Trajectories in Terms of a Kinematic Mapping of Spatial Rigid Motion. ASME J. Mech., Transm., Autom. Des., 109 (1987), 98-100.

[8] O’Neill, B., Semi-Riemannian Geometry with Applications to Relativity, Academic Press, London, 1983.

[9] Ryuh, B. S., Robot trajectory planning using the curvature theory of ruled surfaces. Doctoral dissertation, Purdue University, West Lafayette, Ind, USA, 1989.

[10] Ryuh, B. S., and Pennock, G. R., Accurate Motion of a Robot End-Effector Using the Curvature Theory of Ruled Surface. ASME J. Mech. Transm. Autom. Des., 110 (1988), 383-388.

[11] Roth, B., Finding Geometric Invariants From Time-Based Invariants for Spherical and Spatial Motions. ASME Journal of Mechanical Design, 127 (2005), 227-231.

[12] Stachel, H., Instantaneous Spatial Kinematics and the Invariants of the Axodes, Proceedings Ball 2000 Symposium, Cambridge University Press, London, 23 (2000).

[13] Ting, K. L., Zhang, Y., and Bunduwongse, R., Characterization and Coordination of Point-line Trajectories. ASME Journal of Mechanical Design, 127 (2005), 502-505.

[14] Yücesan, A., Özkan, G., Generalized relaxed elastic line on a non-degenerate surface. International Conference: Mathematical Science and Applications, 26-30 December 2012, Abu Dhabi, UAE. 


\section{Affiliations}

TUNAHAN TURHAN

AdDress: Süleyman Demirel University, Vocational School of Technical Sciences, 32260, Isparta, Turkey. E-MAIL: tunahanturhan@sdu.edu.tr

VILDAN ÖZDEMİR

ADDRESS: Selçuk University, Department of Mathematics, 42250, Konya, Turkey.

E-MAIL: vildanbacak@selcuk.edu.tr

NIHAT AYYILDIZ

ADDRESS: Süleyman Demirel University, Department of Mathematics, 32260, Isparta, Turkey.

E-MAIL: nihatayyildiz@sdu.edu.tr 